\title{
CAPITULO 22 \\ ANÁLISE DOS RISCOS OCUPACIONAIS EM UNIDADES DE ATENÇÃO PRIMÁRIA Á SAÚDE DO INTERIOR DO PIAUÍ FRENTE À PANDEMIA DA COVID19
}

DOI 10.4322/978-65-995353-2-1.c22

\begin{abstract}
Lauana Maria Marques de Oliveira ${ }^{\mathbf{1}}$, Josiane Marques das Chagas ${ }^{2}$, Maria de Jesus de Oliveira França ${ }^{3}$, Nadja Karoline Alves Melo ${ }^{4}$, João Carlos Dias Filho ${ }^{5}$, Jônatas Lucas Marcelino da Silva ${ }^{6}$, Adriana Carvalho Araújo ${ }^{7}$

${ }^{1}$ Enfermeira na Empresa Brasileira de Serviços Hospitalares/ Ebserh, (lauana.oliveira@ebserh.gov.br)

${ }^{2}$ Graduanda em Fisioterapia pela Faculdade de Ensino Superior do Piauí - FAESPI, (josianemarquesdaschagas@gmail.com)

${ }^{3}$ Enfermeira pós graduada em Urgência e Emergência pelo Centro Universitário UNINOVAFAPI, (maria-oliveira83@hotmail.com)

${ }^{4}$ Enfermeira pós graduada em Centro Cirúrgico e Central de Material pelo Centro Universitário UNINOVAFAPI, (nadjakarol@ hotmail.com)

${ }^{5}$ Residente em fisioterapia pela Escola Multicampi de Ciências Médicas - EMCM/UFRJ, (jfilho522@gmail.com)

${ }^{6}$ Graduando em Saúde Coletiva pela Universidade Federal de Pernambuco - UFPE, (jonatas.lucas@ufpe.br) ${ }^{7}$ Enfermeira pós graduada em Unidade de Terapia Intensiva neonatal e pediátrica pela Faculdade Venda Nova do Imigrante - FAVENI, (adriana_carvalhoaraujo@ hotmail.com)
\end{abstract} Resumo

Objetivo: Objetivando analisar os riscos ocupacionais em Unidades de Atenção Primária à Saúde do interior do Piauí frente à pandemia do covid19. Método: traz-se uma pesquisa descritiva e exploratória, com abordagem qualitativa. Os participantes foram os profissionais de saúde destas Unidades, do qual foi realizado uma análise do conteúdo por meio do método de Braun e Clarke, seguindo os aspectos éticos dispostos na Resolução 466/12 do CNS. Participaram desta pesquisa 27 profissionais da saúde, variados entre nível médio, técnico e superior, que citaram diferentes códigos para as ideias das questões apresentadas e estas foram organizadas em mapas. , Resultados: Atualmente, quando se fala em risco de adoecimento, principalmente relacionado a profissionais de saúde em seu ambiente de trabalho, o risco de contágio de covid19 é o mais citado, porém o acidente com pérfuro-cortante é o que mais se destaca. Os locais de trabalho por si já podem comprometer a segurança do trabalhador e seu risco pode ser definido de acordo com a característica da atividade desenvolvida. No contexto da pandemia, muito se tem falado sobre risco de adoecimentos e formas de prevenção nos ambientes de trabalho, já que os profissionais de saúde são a categoria mais exposta, e a exposição é intrínseca à profissão, enfatizando assim a importância dos gestores de saúde como peças fundamentais para a proteção e prevenção dos riscos ocupacionais, e durante a pandemia, diminuir a velocidade de transmissão e achatar a curva de contaminações e mortes, porém a falta de insumos nos ambientes de trabalho ainda é uma das coisas mais citadas. Conclusões: Assim podemos observar que alguns profissionais relacionaram o risco ocupacional exclusivamente ao risco de contrair covid19, mostrando a necessidade de treinamento e a marginalização por parte dos próprios profissionais de saúde a respeito deste tema.

Palavras-chave: Riscos ocupacionais; Atenção primária à saúde; Pandemias. 
Área Temática: Ciências da Saúde.

E-mail do autor principal: lauana.oliveira@ebserh.gov.br

\section{INTRODUÇÃO}

Atualmente, médicos e profissionais da saúde encontram-se perante uma pandemia desafiadora causada por uma nova cepa denominada 2019 Novel Coronavírus (covid-19) da qual causa uma síndrome gripal acompanhada de sintomas respiratórios que podem ser desde leves a graves (NOTA TÉCNICA, 2020).

A China passou a ter transmissão sustentada do Coronavírus em Janeiro de 2020 e, diante desse extenso problema de saúde pública, a Organização Mundial da Saúde (OMS) aumentou o nível de alerta para alto em relação ao risco global do covid19. No Brasil o Ministério da Saúde (MS) confirmou numa quarta-feira (26/2), sendo também o primeiro da América Latina, o primeiro caso de novo coronavírus em São Paulo. Em 03 de fevereiro de 2020 a portaria SVS/MS nº 188 declarou emergência em saúde pública de importância nacional (ESPIN).

Os trabalhadores de saúde, em especial, são os que se encontram mais vulneráveis, e segundo a Nota Técnica: proteção integral a saúde do trabalhador no contexto da pandemia de covid-19 (2020) que, em consonância com a política e as normas de proteção da saúde dos trabalhadores no Brasil, adaptou normas que foram estabelecidas pela Occupational Safety and Health Administration (OSHA) órgão do governo federal americano para proteção da saúde dos trabalhadores e classificou os trabalhadores sob perigo de exposição.

Em todo o mundo há uma grande contaminação e adoecimento dos profissionais envolvidos no atendimento aos pacientes acometidos por covid19 (WANG; ZHOU; LIU, 2020). No Brasil o maior número de profissionais acometidos dentro desta categoria é o de nível médio (RAFAEL; NETO; CARVALHO; ET. AL., 2020). Vale ainda lembrar que a concentração de profissionais é tradicionalmente maior nos grandes centros urbanos brasileiros, este cenário complexo impõe desafios adicionais à vigilância epidemiológica, às relações internacionais e à programação de políticas públicas fazendo com que assim haja desigualdade no acesso aos sistemas de saúde e precárias condições de autocuidado.

\section{MÉTODO}

A pesquisa foi realizada de forma descritiva e exploratória, com abordagem qualitativa, desenvolvida nas Unidades Básicas de Saúdes (UBS) e Unidade Mista de Saúde (UMS), de caráter público, situado na cidade de Lagoa Alegre/PI, Brasil. Os participantes incluídos nesse estudo foram todos os profissionais de saúde das Unidades, de ensino fundamental, médio e superior, que estavam regulamente contratados e locados nas Unidades selecionadas. Para esta 
pesquisa foi definido como profissionais de saúdes todos aqueles que estavam devidamente lotados em uma das Unidades da pesquisa e prestando cuidados diretos ao público. Serão excluídos os profissionais que não forem lotadas nas Unidades selecionadas, contratados e/ou concursados e que estejam sob regime de prestação de serviços temporários, ou aqueles que apresentarem-se de férias ou afastados.

A seleção dos participantes ocorreu considerando a disponibilidade e o interesse dos mesmos em participarem da pesquisa, por meio da assinatura do Termo de Consentimento Livre e Esclarecido (TCLE), o qual por sua vez, obedece aos aspectos éticos e legais, conforme determinação da Resolução no 466/12. A análise do conteúdo se dará por meio do método de Braun e Clarke (2006) que sintetizam a Análise temática (AT) em seis etapas: familiarização com dados; gerando códigos iniciais; buscando temas; revisando os temas; definindo e nomeando os temas e produzindo o relatório.

O projeto foi submetido a um Comitê de Ética para a obtenção do conhecimento do conteúdo científico-metodológico desse estudo, por meio da CAAE: 36621020.6.0000.5209. A Secretaria Municipal de Saúde das Instituições escolhidas, comunicada do desejo de realização da pesquisa via ofício, como instituição Co-Participante assinou termo de consentimento favorável.

\section{RESULTADOS E DISCUSSÃO}

Participaram desta pesquisa 27 profissionais da saúde, 19 do sexo feminino e 08 do sexo masculino, destes 07 eram do nível médio, 09 do nível técnico e 11 do nível superior. A idade predominante foi 20-29 anos, 20 participantes se consideram pardos/mulatos, 21 eram da religião católica, a maioria dos entrevistados tinham renda de 01-02 salários mínimos, 18 tinham de 3-5 pessoas morando em casa, 10 participantes eram casados e também 10 eram solteiros e cerca de 14 participantes moravam na cidade de Lagoa Alegre.

Fonseca et. al. (2020), numa perspectiva inovadora, define o risco ocupacional como uma probabilidade de algum elemento ou circunstancia no ambiente de trabalho que traga prejuízos ao trabalhador, tais riscos podem ser observados como contaminações, lesões ou quais outros prejuízos a saúde física e mental dos trabalhadores. Durante os questionamentos muitos participantes citaram diversos riscos de adoecimentos ou contaminações em seus ambientes de trabalho, definido o risco ocupacional como um risco que está presente dentro e fora do ambiente de trabalho, que há risco mesmo com o uso de Equipamentos de Proteção Individual (EPI) ou coletiva (EPC), que a falta de atenção pode contribuir e que varia de acordo com o setor de lotação.

Então, ao analisar as entrevistas e considerando os objetivos propostos neste estudo,

E - book Tripé do Ensino Superior: Ensino, Pesquisa e Extensão 
foram citadas pelos participantes diferentes códigos para as ideias das questões apresentadas, que foram organizadas em mapas (figuras de 01 a 04) e um gráfico, de acordo com o proposto por Braun e Clarke (2006). A figura 01 traz a ilustração do que representa os riscos ocupacionais nestas Unidades:

Figura 01: O que é risco ocupacional?

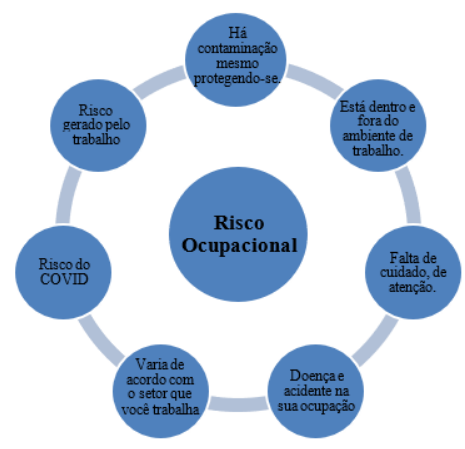

Figura 01: Autores, 2021.

Foi citado ainda o risco de adoecimentos ou lesões, assim como o risco de adoecimento ou contágio de covid19. É evidente que os ambientes de trabalho por si já podem comprometer a segurança do trabalhador e o risco dele pode ser definido de acordo com a característica da atividade desenvolvida. Muito tem-se questionado sobre o risco de adoecimento por covid19 durante as jornadas de trabalho ou a possível contaminação durante o trajeto até o trabalho e a OMS (2019) em seu Plano de resposta à COVID-19 e prontidão estratégica traz três objetivos principais, contando com a proteção a saúde dos trabalhadores e a segurança aos pacientes em seu terceiro objetivo.

Além dos riscos de contaminação por covid19, as falas nos trazem os riscos intrínsecos à profissão, aqueles que podem acontecer mesmo com meios de proteção adequados, neste quesito podemos citar o acidente com pérfuro-cortante, que é o risco ocupacional que mais se destaca relacionado a profissionais de saúde, principalmente na categoria médica e de enfermagem (GOULART; ROCHA; CARVALHO; ET. AL., 2020), o que traz grandes prejuízos ao trabalhador contando que este se expõe a fluídos biológicos.

Muitos tem se falado sobre a utilização de EPI durante a pandemia e este foi o relato mais citado para o desencadeamento de riscos ocupacionais, já que os profissionais de saúde são a categoria mais exposta a este risco dentro dos ambientes de trabalho, a covid19 é altamente contagiosa e não há como evitar a exposição destes profissionais, já que em alguns casos estes trabalhadores fazem ainda o atendimento direto ao paciente suspeito (VEGAL; ANTONIOLLI; MACEDO; ET. AL, 2021), por isso estes profissionais foram questionados sobre o que poderia desencadear riscos ocupacionais no seu ambiente de trabalho e o que poderia ser feito por eles

\section{E - book Tripé do Ensino Superior: Ensino,} Pesquisa e Extensão 
para a prevenção destes riscos, dos quais as falas citadas foram ilustradas na figura 02 e 03 :

Figura 02: O que desencadeia riscos ocupacionais?

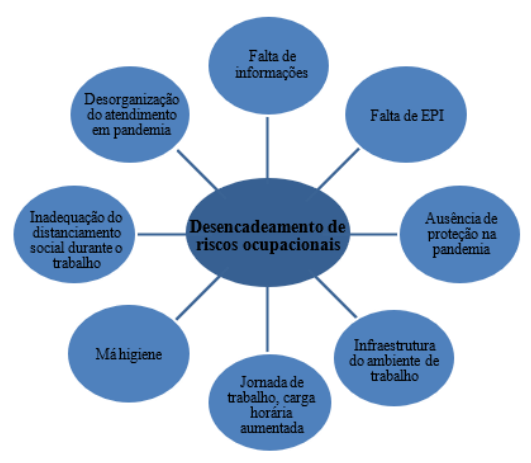

Figura 02: Autores, 2021.
Figura 03: O que pode ser feito para previnir tais riscos?

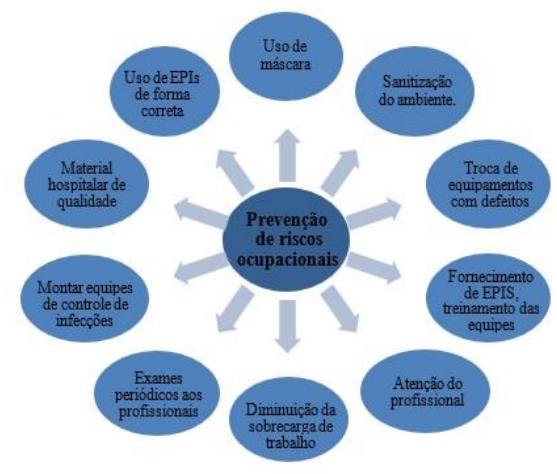

Figura 03: Autores, 2021.

Um dos grandes desafios também enfrentados foi a escassez de EPI, já que mesmo após a pandemia de SARS de 2003 os serviços de saúde não conseguiram se organizar (BERLINGER; WYNIA; POWELL; ET. AL., 2020) para uma futura escassez e alta demanda de equipamentos. Mas somente o uso destes equipamentos não isenta estes profissionais dos riscos ou contaminações, Liu et. al. (2020) relatam que a lavagem das mãos inadequadas de profissionais que atendem pacientes acometidos por covid19 traz um risco maior de contrair a contaminação.

A falta de informações da população em geral e a difícil missão de lidar com algo desconhecido também gerava pavor, a falta de investimento em treinamentos adequados pode ter influenciado na falta de destreza por partes dos profissionais. Arons et. al. (2020) traz que a possível contaminação dos profissionais possa ocorrer nas falhas de colocação ou retirada de EPI, o que é confirmado por Berlinger et. al. (2020) quando mostra que cerca de $90 \%$ dos profissionais não sabiam seguir a sequência ou técnica correta de colocação ou retirada dos EPIs. O tempo de transmissibilidade do vírus com o paciente ainda assintomático também pode contribuir para a infecção (ARONS; HATFIELD; REDDY; at. Al., 2020).

É notável que a alta carga de trabalho pode ainda atrapalhar no desempenho de atividades de proteção ocupacional dos profissionais, os da saúde costumam atuar em jornadas duplas, com equipes reduzidas, onde há má qualidade de equipamentos por insuficiência ou falta de investimentos, que expõem profissionais a riscos e desmotiva as equipes (PORTO; MARZIALE, 2016).

O cenário pandêmico vivenciado pelos profissionais traz riscos novos, com a 
necessidade de identificação destes riscos, mudanças de hábitos e resolução de novas questões, muitos identificam dificuldades não enfrentadas antes, uso de novos recursos, utilizar materiais que não estão disponíveis (ALMEIDA, 2020) e quando se fala em prevenção de riscos ocupacionais, o uso da máscara tanto por parte de profissionais, como dos próprios pacientes neste cenário de pandemia tem sido a fala mais constante.

Ao se tratar de trabalhadores que atendem pacientes críticos, esse risco está relacionado à assistência ao paciente e procedimentos geradores de aerossóis (LIU; HE; HUIGUO; ET. AL., 2020) o que retoma a importância dos EPI como instrumentos intrínsecos a jornada de trabalho, já que a consolidação dos relatos basicamente nos retoma a prevenção de adoecimentos por covid19 associado ao risco ocupacional.

Messias et. al. (2020) nos traz a susceptibilidade dos profissionais de saúde devido a sua atuação direta aos pacientes acometidos por covid19 e a pressão psicológica tem sido algo evidenciado por parte destes profissionais e seus familiares. A higienização dos ambientes, fornecimentos de insumos, redução da carga de trabalho e treinamento dos profissionais foram opções citadas que poderiam amenizar estes riscos ou até mesmo trazer mais segurança e bem estar aos profissionais envolvidos nesta realidade.

Reda, Fisseha, Mengistie, et. al. (2015) reforçam a ideia de que as normas e rotinas bem estabelecidas adequam as condições de trabalho. É importante que profissionais tenham consciência de seus papéis nas prevenções de riscos ocupacionais, a definição de fluxos e a divulgação destes traz conhecimento aos trabalhadores e os empenha a agir de forma correta diante destas exposições. Tais profissionais foram questionados ainda a respeito do papel dos seus gestores na amenização dos riscos ocupacionais aos quais está exposto em seu ambiente de trabalho e os relatos foram evidenciados pela figura 04 :

Figura 04: O que os gestores da saúde poreriam fazer para reveter os riscos ocupacionais?

Figura 04: Autores, 2021.

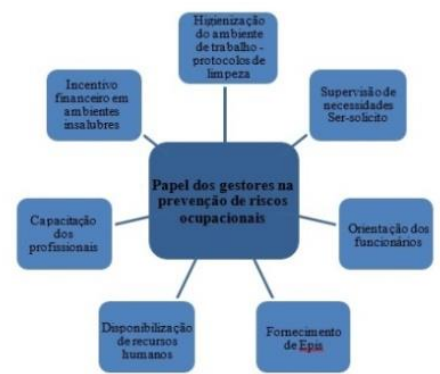

Os gestores de saúde são peças fundamentais para a proteção e prevenção dos riscos ocupacionais, durante a pandemia, diminuir a velocidade de transmissão e achatar a curva de contaminações e mortes têm sido um papel fundamental (ALMEIDA, 2020). A preocupação dos profissionais com a higienização do ambiente e a implementação de protocolos de limpeza

E - book Tripé do Ensino Superior: Ensino, Pesquisa e Extensão 
da Unidade só reforçam a preocupação dos profissionais com a possibilidade de contaminações em seu local de trabalho.

A necessidade de os gestores reconhecerem as necessidades da Unidade e estarem presentes também evidencia uma difícil realidade em nosso cenário, onde quem está na ponta dificilmente conhece as necessidades instaladas. Balthazar et. al. (2017) diz que a responsabilidade dos gestores deve dar atenção especial às obrigações legais, também traz programas de treinamentos e utilização de EPIs como imprescindível a sua atuação.

A falta de recursos humanos também tem sido discutida, assim como a falta de incentivo financeiro e carga horária exaustiva. O Senado Federal por meio do Projeto de Lei № 1802 de 2020, indica a insalubridade trabalhista no valor de $40 \%$ do salário mínimo aos profissionais que estão expostos ao covid19 do setor público e privado.

Podemos observar ainda que pode ter sido evidenciado falhas na comunicação diante do dialogo gestor-trabalhador, trazendo insatisfação a respeito dos riscos ocupacionais diante do cenário que estão expostos e baixas medidas de prevenção existentes, sendo assim, os profissionais foram ainda questionados sobre a utilização de EPI e EPC em seu ambiente de trabalho, a presença de informativos sobre os riscos existentes nas Unidades, a presença de treinamentos e capacitações sobre como preveni-los, que foram ilustrados através de gráfico:

Gráfico 01: A utilização de EPIs, EPCs, treinamentos e disponibilização de inofrmativos nas Unidades de trabalho.

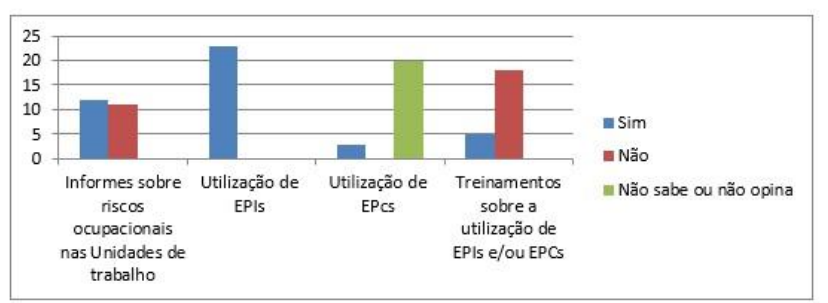

Gráfico 01: Autores, 2021.

Os relatos sobre o recebimento de informes sobre os riscos ocupacionais existentes nas Unidades estão semelhantes, nos trazendo a ideia de que os profissionais de saúde são informados sobre os riscos, porem quando comparamos a utilização de EPIs e EPCs por parte dos profissionais a diferença é estonteante, boa parte dos profissionais responderam que não utilizam EPI coletivo, ou seja, não sabem definir o que seja EPC.

A Fiocruz define EPC como equipamentos utilizados para eliminar ou agentes de risco, protegendo vários indivíduos simultaneamente e reduzindo as consequências em caso de acidentes. Os participantes citaram ainda a utilização apenas de EPI esquecendo os EPCs, ratificando ainda mais o exposto na evidencia anterior. Quando foram indagados sobre os treinamentos sobre a utilização dos meios de proteção, uma pequena parcela apenas cita que recebeu treinamento, e estes foram ainda realizados pela enfermeira da sua própria equipe, 
trazendo aqui a enfermagem como protagonista da equipe de Estratégia de Saúde da Família (ESF).

Podemos observar então que o trabalho desenvolvido tange quase que exclusivamente ao assistencialismo deixando de lado a saúde pessoal por parte do próprio trabalhador, alguns apresentam falta de conhecimento, outros, falta de incentivo por parte das próprias Unidades em capacitação e incentivo de desempenho a estes trabalhadores. Machado (2020) mostra que profissionais de saúde têm aumentado a sua carga de trabalho e possuem salários baixos se comparados ao cenário internacional.

A eficácia dos EPIs está relacionada ao fornecimento adequado, treinamentos e uso correto e consciente. O uso racional requer tanto o papel dos gestores em capacitar seus funcionários como dos próprios funcionários em reconhecer as necessidades e utiliza-los de forma adequada. Gallasch et. al. (2020) relata que a legislação trabalhista brasileira, NR32, traz a obrigatoriedade do provimento de EPIs por parte do empregador, e o compromisso do empregado em manter e zelar por ele.

\section{CONCLUSÃO}

Diante do exposto podemos concluir que a maioria dos profissionais consegue definir risco ocupacional, porém apresentam baixo conhecimento dos riscos que estão expostos em suas Unidades de trabalho, assim como as medidas de proteção e prevenção, atrelando o risco de adoecimento, quase que em sua totalidade, ao risco do acometimento pela covid19, observando assim que pode haver uma marginalização por parte dos próprios profissionais de saúde a respeito deste tema, trazendo uma necessidade de treinamento. No tocante a responsabilidade dos gestores, é interessante citar que os participantes sentem a ausência destes e que a falta de insumos e recursos humanos muito pode atrapalhar o desempenho do trabalhador assim como o risco de acometimento no meio ocupacional.

Tendo em vista a baixa quantidade de estudos relacionados a saúde ocupacional na Atenção Básica ( $\mathrm{AB}$ ) em tempo de pandemia, as indagações sobre esses riscos, maneiras de prevenção e proteção, assim como o papel dos gestores na saúde dos trabalhadores, sugere-se o desenvolvimento de outros estudos que possam evidenciar a necessidade de intervenções que possibilite uma construção maior arranjada no bem estar dos profissionais de saúde brasileiros.

\section{REFERÊNCIAS}

AGÊNCIA NACIONAL DE VIGILÂNCIA SANITÁRIA - ANVISA. Nota Técnica GVIMS/GGTES/ANVISA Nº 04/2020: orientações para serviços de saúde: medidas de prevenção e controle que devem ser adotadas durante a assistência aos casos suspeitos ou confirmados de infecção pelo novo coronavírus (sars-cov-2).

\section{E - book Tripé do Ensino Superior: Ensino, Pesquisa e Extensão}


ALMEIDA, I. M. Proteção para a saúde dos trabalhadores da saúde em tempos de COVID-19 e respostas à pandemia. Health protection for healthcare workers in CPVID19 times and responses to the pandemic. Botucatu, SP: Dossiê COVID-19 e Saúde do Trabalhador/Ensaio. Rev. bras. saúde ocup. 45, 2020.

ARONS, M. M. et. al. Presymptomatic SARS-CoV-2 infections and transmission in a skilled nursing facility. 382: 2081-2090. Massachusetts: N Engl J Med, 2020. 382:2081-90.

BALTHAZAR, M. A. P. et. al. Gestão dos riscos ocupacionais nos serviços hospitalares: uma análise reflexiva. v. 11, n. 9. Recife: Revista de enfermagem, 2017.

BERLINGER, N. et. al. Ethical framework for health care institutions responding to novel coronavirus SARSCoV-2 (COVID-19):guidelines for institutional ethics services responding to COVID-19: managing uncertainty, safeguarding communities, guiding practice. Garrison: The Hastings Center, 2020.

BRASIL, MINISTÉRIO DA SAÚDE. Diretrizes e normas regulamentadoras de pesquisas. Conselho Nacional de Saúde. Resolução no 466, de 12 de dezembro de 2012.

BRASIL. MINISTÉRIO DO TRABALHO E EMPREGO. Portaria nş 485, de 11 de novembro de 2005. Aprova a norma regulamentadora nş 32 (Segurança e saúde no trabalho em estabelecimentos de saúde) Diário Oficial da República Federativa do Brasil,

Brasília(DF), 2005. Nov 11.

BRAUN, V.; CLARKE, V. Using thematic analysis in psychology. Qualitative Research in Psychology. v. (2006). Washington: American Psychological Association, 2006. 3(2), 77101.

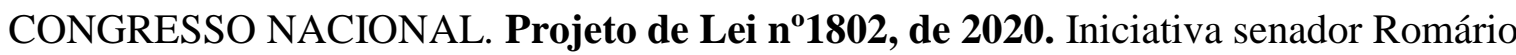
(PODEMOS/RJ). Assunto Social - Saúde. Natureza: Norma Geral.

COSTA, D. F. et. al. Nota técnica: proteção integral a saúde do trabalhador no contexto da pandemia de covid-19 (sars-cov-2). João Pessoa: DPS/CCM-NESC/CCS-UFPB 2LASAT/IAM, 2020.

FONSECA, E. C. et. al. Riscos ocupacionais na sala de vacinação e suas implicações à saúde do trabalhador de enfermagem. v. 28. Rio de Janeiro: Rev. enferm. UERJ, 2020. 28: e45920.

FONTANELLA, B. J. B. et al. Amostragem em pesquisas qualitativas: proposta de procedimentos para constatar saturação teórica. vol.27. Rio de Janeiro: Cad. Saúde Pública, 2011. no.2, p. 389-394.

FUNDAÇÃO OSWALDO CRUZ- FIOCRUZ - NUBI. Núcleo de biossegurança. EPCEQUIPAMENTO DE PROTEÇÃO COLETIVA. Disponível em:http://biosseguranca.ensp.fiocruz.br/equipamentos-de-protecao/epc. Acesso em: $11 \mathrm{de}$ Agosto de 2021.

GALLASCH, C. H. et. al. Prevenção relacionada à exposição ocupacional do profissional de saúde no cenário de COVID-19. v. 28. Rio de Janeiro: Rev. enferm. UERJ, 2020. 28 : 49596. 
LIU, M. et. al. Clinical characteristics of 30 medical workers infected with new coronavirus pneumonia. 17;43(0):E016. Chinese, 2020. 43(3):209-14.

MACHADO, M. H. Os Profissionais de saúde em tempos de COVID19 - a realidade brasileira. ). Observatório Covid-19 Fiocruz, 2020. 3p.

MESSIAS, J. M. A.; SILVA, S. C. C.; PRADO, I. F. A covid-19 e os riscos ocupacionais para profissionais de enfermagem. $v$. 30. Fragmentos de cultura: Revista Interdisciplinar de Ciências Humanas - Interdisciplinary Journal of Human Sciences, 2020. N. 04.

MINISTÉRIO DA SAÚDE/GM. Portaria N 188, de 3 de Fevereiro de 2020. Diário Oficial da União, publicado em: 04/02/202 | Edição: 24-A | Seção: 1 - Extra | Página: 1.

PORTO, J. S.; MARZIALE, M. H. P. Motivos e consequências da baixa adesão as precauções padrão pela equipe de enfermagem. (2016) 37(2):e57395x. Rev. Gaúcha Enferm. Doi: https://doi.org/10.1590/1983-1447.2016.02.57395

RAFAEL, R. M. R. et. al. Epidemiologia, políticas públicas e pandemia de Covid-19: o que esperar no Brasil? v. 28. Rio de Janeiro: Rev enferm UERJ, 2020. 28:e49570.

REDA, A. A. et. al. Standard precautions: ocupacional exposure and behavior of health care workers in ethiopia. Srikanth Tripathy, National AIDS Research Institute. Índía: PLoS One, 2010. 4];5(12):e14420. http://dx.doi.org/10.1371/journal.pone.0014420

VEJA, E. A. U. et. al. Riscos de adoecimento ocupacional em profissionais da saúde que atenem pacientes com covid-19: revisão integrativa. v. 29. Rev. Latino-Am. Enfermagem, 2021. 29:e3455. www.eerp.usp.br/rlae.

WANG, J.; ZHOU, M.; LIU, F. Exploring the reasons for healthcare workers infected with novel coronavirus disease 2019 (COVID-19) in China. Edição 1. The Healthcare Infection Society. Published by Elsevier Ltd, 2020. Vol 105, p100-101. 